\title{
Mid-infrared interferometry of the massive young stellar object NGC 2264 IRS $1^{\star}$
}

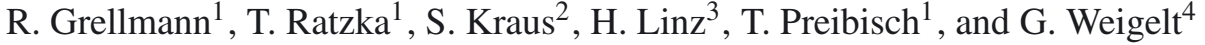 \\ ${ }^{1}$ Universitäts-Sternwarte, Ludwig-Maximilians-Universität München, Scheinerstr. 1, 81679 München, Germany \\ e-mail: grellman@usm.uni-muenchen.de \\ 2 University of Michigan, Department of Astronomy, Ann Arbor, MI 48109-1090, USA \\ 3 Max-Planck-Institut für Astronomie, Königstuhl 17, 69117 Heidelberg, Germany \\ ${ }^{4}$ Max-Planck-Institut für Radioastronomie, Auf dem Hügel 69, 53121 Bonn, Germany \\ Received 11 February 2011 / Accepted 22 June 2011
}

ABSTRACT

\begin{abstract}
Context. The optically invisible infrared-source NGC 2264 IRS 1 lying north of the Cone Nebula is thought to be a massive young stellar object $\left(\sim 10 M_{\odot}\right)$. Although strong infrared excess clearly shows that the central object is surrounded by large amounts of circumstellar material, no information about the spatial distribution of this circumstellar material has been available until now.

Aims. We used the ESO Very Large Telescope Interferometer to perform long-baseline interferometric observations of NGC 2264 IRS 1 in the mid-infrared regime. Our observations resolve the circumstellar material around NGC 2264 IRS 1 , provide the first direct measurement of the angular size of the mid-infrared emission, and yield direct constraints on the spatial distribution of the dust.

Methods. We analyze the spectrally dispersed interferometric data taken with MIDI at two different position angles and baseline lengths. We use different approaches (a geometrical model, a temperature-gradient model, and radiative transfer models) to jointly model the observed interferometric visibilities and the spectral energy distribution.

Results. The derived visibility values between $\sim 0.02$ and $\sim 0.3$ show that the mid-infrared emission is clearly resolved. The characteristic size of the MIR-emission region is $\sim 30-60 \mathrm{AU}$; this value is typical for other YSOs with similar or somewhat lower luminosities. A comparison of the sizes for the two position angles shows a significant elongation of the dust distribution. Simple spherical envelope models are therefore inconsistent with the data. The radiative transfer modeling of our data suggests that we observe a geometrically thin and optically thick circumstellar disk with a mass of about $0.1 M_{\odot}$.

Conclusions. Our modeling indicates that NGC 2264 IRS 1 is surrounded by a flat circumstellar disk that has properties similar to disks typically found around lower-mass young stellar objects. This result supports the assumption that massive young stellar objects form via accretion from circumstellar disks.
\end{abstract}

Key words. techniques: interferometric - stars: individual: NGC 2264 IRS 1 - stars: formation - circumstellar matter

\section{Introduction}

The question of how massive stars $\left(>8 M_{\odot}\right.$ ) form remains unclear because observations of massive young stellar objects (MYSOs) are rare (Zinnecker \& Yorke 2007). This has several reasons. First of all, these objects are surrounded by large amounts of gas and dust, which absorb nearly all the optical and near-infrared (NIR) radiation from the central source, thus making a direct observation very difficult. Second, their large distances mean that to study them we need data of high angular resolution. Other difficulties are the fast evolution of MYSOs and that they are rarely found in isolation.

Two different formation scenarios for MYSOs are currently discussed: the coalescence scenario and a formation similar to low mass protostars. In the first scenario, several low mass stars merge and finally form a single more massive star (Bonnell et al. 1998; Zinnecker \& Bate 2002). In the second scenario, the radiation pressure problem that would prevent spherical accretion as soon as the protostellar mass exceeds $\sim 10 M_{\odot}$ is solved by stellar outflows, which open a cavity in polar direction, through which the radiation can escape and the radiation pressure in the equatorial plane is lowered. Therefore, an infall of material in this plane

\footnotetext{
* Based on observations collected at the European Organisation for Astronomical Research in the Southern Hemisphere, Chile, observing programs 076.C-0725(B) and 082.C-899(A).
}

is possible. Observations have provided some evidence that massive stars can form in an analogous way to low mass stars. For instance, circumstellar disks (e.g., IRAS 20126+4104, Cesaroni et al. 2005; AFGL 490, Schreyer et al. 2006; IRAS 13481-6124, Kraus et al. 2010), molecular outflows, or collimated jets (e.g., Cunningham et al. 2009) are often interpreted as evidence of ongoing accretion. From the theoretical side, both the competitive accretion (Bonnell \& Bate 2006) and the turbulent core model (McKee \& Tan 2003) agree with the formation of disks. Furthermore, simulations demonstrate that high-mass stars may form by disk accretion (Kuiper et al. 2010).

Traditionally, the spatial distribution of the surrounding dust was usually studied by modeling the spectral energy distribution (SED). Such SED model fits are highly ambiguous (Thamm et al. 1994; Men'shchikov \& Henning 1997). Therefore, more information is required to determine the real geometry of the circumstellar material. To test the existence of a circumstellar disk, and thus the disk accretion scenario of massive star formation, information at high angular resolution (corresponding to physical scales below $100 \mathrm{AU}$ ) is needed. Furthermore, only radiation at long wavelengths (e.g., infrared, sub-mm) is able to escape from the dense dusty surroundings of the star. Infrared long-baseline interferometry can thus provide direct spatial information on the required small scales. 
NGC 2264 is a young, star-forming cluster with an age of about 3 Myr located in the Mon OB 1 molecular cloud complex. Literature values for the distance of the cluster vary between $400 \mathrm{pc}$ and $1000 \mathrm{pc}$, although here we adopt the most recent value of 913 pc (Baxter et al. 2009). NGC 2264 IRS 1, the brightest IR-source in this region, was discovered in 1972 by Allen (hence is also called Allen's Source) and has no optical counterpart. Allen (1972) pointed out that since NGC 2264 IRS 1 is the brightest and most luminous source in this field, it is the likely source of the radiation pressure creating the Cone Nebula. It is believed to be a young star with a mass of $9.5 M_{\odot}$, spectral type B0-B2 (Thompson et al. 1998), a luminosity of $3.5 \times 10^{3} L_{\odot}$ (Harvey et al. 1977), and a visual extinction of 20-30 mag. However, owing to its high extinction in the optical, all values are rather uncertain and should be interpreted with care, e.g., luminosity values between $2.3 \times 10^{3} L_{\odot}$ (Nakano et al. 2003) and $4.7 \times 10^{3} L_{\odot}$ (Schwartz et al. 1985) can be found in the literature.

NGC 2264 IRS 1 is associated with molecular outflows and dense molecular clumps, and in NIR images a twisted jet-like feature can be seen extending north of the source (Schreyer et al. 1997; Ward-Thompson et al. 2000). The morphology of the circumstellar environment is unclear. Several studies did not find any hint of an asymmetric structure. Schreyer et al. (2003) were unable to detect any signs of a circumstellar disk in their molecular line study and de Wit et al. (2009) do not discern any disklike structure in their images taken in the MIR with COMICS. However, models of a spherical symmetric distribution of the circumstellar material fail to reproduce the SED of the object in its entirety and were ruled out in several publications (Thompson \& Tokunaga 1978; Harvey et al. 1977). However, the spatial resolution of all these studies may not be sufficient to resolve a disk-like structure.

In this paper, we present mid-infrared interferometric observations of the MYSO NGC 2264 IRS 1 performed with the MidInfrared Interferometric Instrument (MIDI) at the Very Large Telescope Interferometer (VLTI). The MIDI data and their reduction are shown in Sect. 2. Different modeling approaches (geometrical, temperature-gradient, and radiative transfer models) for the visibilities and the SED are presented in Sect. 3. Implications for the object and final conclusions are discussed in Sect. 4.

\section{Observations and data reduction}

NGC 2264 IRS 1 was observed on 2005 December 23 in the course of ESO observing programme 076.C-0725(B) (PI: Kraus) and on 2009 February 15 in the course of ESO programme 082.C-0899(A) (PI: Feldt) with MIDI at the VLTI (see Table 2). MIDI (Leinert et al. 2003) is a two-telescope beam-combiner working in the wavelength range from 8 to $13 \mu \mathrm{m}$ and using the principle of a Michelson interferometer. To measure the fringe amplitude, the optical path difference (OPD) is varied with internal delay lines. The 2005 data of NGC 2264 IRS 1 were taken with a baseline of $89.1 \mathrm{~m}$ in the SCI-PHOT mode, in which interferometric and photometric data are measured simultaneously. The grism (a combination of a grating and a prism) with a spectral resolution of $\frac{\Delta \lambda}{\lambda}=230$ was used. In the same night, two calibrators were observed with the same configuration, HD 39425 a few hours earlier and HD 61421 directly after the object. The 2009 data were taken with a baseline of $40.2 \mathrm{~m}$ in the HIGHSENS mode, in which interferometric and photometric data are obtained separately. For this observation, the prism with a spectral resolution of $\frac{\Delta \lambda}{\lambda}=30$ was used. HD 48217 and HD 49161 served as calibrators.
Table 1. Measured fluxes for NGC 2264 IRS 1.

\begin{tabular}{lccc}
\hline \hline $\begin{array}{l}\text { Wavelength } \\
{[\mu \mathrm{m}]}\end{array}$ & $\begin{array}{c}\text { Flux } \\
{[\mathrm{Jy}]}\end{array}$ & Instrument/Reference & Aperture \\
\hline $2-45$ & $9-670$ & ISO (Sloan et al. (2003)) & $14^{\prime \prime} \times 20^{\prime \prime}$ \\
1.65 & $0.9 \pm 0.04$ & 2-MASS & $4^{\prime \prime}$ \\
2.2 & $6.8 \pm 0.016$ & 2-MASS & $4^{\prime \prime}$ \\
$8-13$ & $60-130$ & MIDI & $0.5^{\prime \prime}$ \\
12.0 & $146 \pm 9$ & IRAS & $0.75^{\prime} \times 4.5^{\prime}$ \\
24.5 & 330 & COMICS & $42^{\prime \prime} \times 32^{\prime \prime}$ \\
25.0 & $324 \pm 13$ & IRAS & $0.75^{\prime} \times 4.6^{\prime}$ \\
53.0 & $980 \pm 50$ & Harvey et al. (1977) & $17^{\prime \prime}$ \\
60.0 & 911 & IRAS & $1.5^{\prime} \times 4.7^{\prime}$ \\
70.0 & $960 \pm 290$ & Sargent et al. (1984) & $3^{\prime}$ \\
100.0 & 1560 & IRAS & $3^{\prime} \times 5^{\prime}$ \\
100.0 & $1645 \pm 82$ & Harvey et al. (1977) & $28^{\prime \prime}$ \\
175.0 & $1530 \pm 77$ & Harvey et al. (1977) & $46^{\prime \prime}$ \\
350.0 & $188 \pm 70$ & Chini et al. (1986) & $30^{\prime \prime}$ \\
1300 & $13 \pm 2.5$ & Chini et al. (1986) & $90^{\prime \prime}$ \\
\hline
\end{tabular}

Table 2. Observation $\log$ of the MIDI measurements of NGC 2264 IRS 1.

\begin{tabular}{lccc}
\hline \hline Date & $\begin{array}{c}\text { Proj. Baseline } \\
{[\mathrm{m}]}\end{array}$ & $\begin{array}{c}\text { PA } \\
{\left[{ }^{\circ}\right]}\end{array}$ & Mode \\
\hline $12 / 23 / 2005$ & 89.1 (UT2-UT4) & 81.1 & SCI-PHOT\&GRISM \\
$02 / 15 / 2009$ & 40.2 (UT2-UT3) & 43.9 & HIGH-SENS\&PRISM \\
\hline
\end{tabular}

For data reduction and calibration, we used the software package MIA+EWS (Köhler \& Jaffe 2008). With this software, the correlated flux, visibility, and the total flux can be determined. Typical errors in one single MIDI visibility measurement are about 10\% (Leinert et al. 2004). As an estimate of the error bars, the standard deviation in the results for different calibrators was used. As the conditions during the observation in 2009 were not photometric, we did not use the 2009 photometry data, but the photometry from the 2005 measurement to obtain the visibility. Figure 1 (left) shows the calibrated visibilities for both observations.

Furthermore, a low-resolution IR spectrum of NGC 2264 IRS 1, measured with the SWS instrument aboard the ISO satellite is available (TDT No. 71602619, PI: Whittet), as well as fluxes in several other wavelength bands. These data points are listed in Table 1. Figure 1 (right) shows the total flux spectrum of NGC 2264 IRS 1 as measured by MIDI in 2005 compared to the total flux spectrum measured by the ISO satellite. Both spectra display a deep absorption feature over the whole MIDI wavelength range caused by silicates. The shape of both spectra (MIDI and ISO) is similar but the flux level of the MIDI spectrum is about 25 Jy (about 20\%) lower, which might be due to the different beam sizes of the instruments (see Table 1). The spurious feature in the MIDI spectrum at wavelengths between $9.3 \mu \mathrm{m}$ and $9.8 \mu \mathrm{m}$ is due to atmospheric ozone. Several absorption features can be identified in the ISO spectrum (Gibb et al. 2004). The broad absorptions around 3.1 and $6.0 \mu \mathrm{m}$ arise mainly from water ice, while the carrier of the $6.8 \mu \mathrm{m}$ feature is more uncertain, but might be associated with solid $\mathrm{NH}_{4}^{+}$. A rather sharp feature at $4.27 \mu \mathrm{m}$ is commonly attributed to $\mathrm{CO}_{2}$ ice (Guertler et al. 1996), as is the absorption seen at $15.2 \mu \mathrm{m}$. The feature around $4.7 \mu \mathrm{m}$ is probably a combination of $\mathrm{CO}$ gas line absorption and a variety of ice absorption features. All these findings point to relatively large column densities of cold dense material along the line of sight towards the central source. 

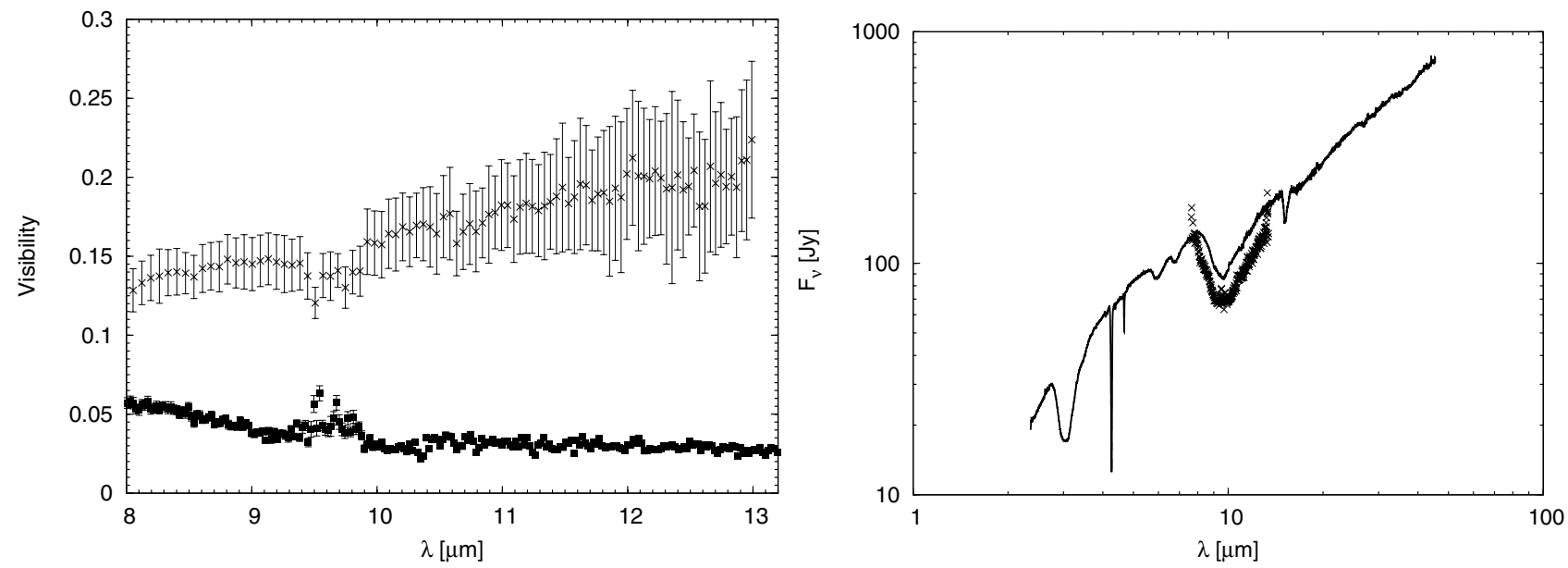

Fig. 1. Left: visibilities of NGC 2264 IRS 1 measured with MIDI. The visibility points measured in 2005 (projected baseline length $89.1 \mathrm{~m}$ ) are shown as rectangulars, those measured in 2009 (projected baseline $40.2 \mathrm{~m}$ ) are shown as crosses. Right: spectrum of NGC 2264 IRS 1 as measured with MIDI (black crosses) in 2005 compared to the ISO spectrum (black line).

\section{Modeling}

We now attempt to derive the properties of NGC 2264 IRS 1 from our measurements using different modeling approaches. First, we use simple geometrical models to characterize the size of the dust emission region (see Sect. 3.1). To test for the possibility of a circumstellar disk, we compare the data with a temperature-gradient model for such a disk (Sect. 3.2). To check the parameter space over a wider range, we employ the online model SED fitter by Robitaille et al. (2007) in Sect. 3.3. A more detailed modeling is done in Sect. 3.4, where the twodimensional radiative transfer code RADMC is used.

\subsection{Simple geometrical models}

As a first step, one can derive an estimate of the diameter of the mid-infrared (MIR) emission region of the object from the visibilities by using simple geometrical models. For more details of this we refer to Berger \& Segransan (2007) and Tristram (2007).

We first consider the possibility of a binary. Its existence would result in a sinusoidal variation in the visibility (versus wavelength), ranging between a maximum and a minimum value. The maximum value depends on the extension of the MIR emission regions (and would be 1 for point like sources), whereas the amplitude depends on the flux ratio of both components (the minimum would be 0 for a flux ratio of $1: 1$ ). Owing to the slit width of MIDI (see Table 1), the maximum separation of a binary that can be identified with this instrument is $\sim 230 \mathrm{AU}$. The minimum separation at which a binary could be resolved with the $90 \mathrm{~m}$ baseline is $\sim 30 \mathrm{AU}$ (diffraction limit; half a period of the modulation in the visibility would be seen). Both observed visibilities show no typical sinusoidal variation, hence a binary system with a separation of $>30 \mathrm{AU}$ is unlikely and not considered during the modeling process. However, with our measurements we could miss very faint binary companions or systems with separations of about $30 \mathrm{AU}$ oriented non-parallel to the position angle of the $90 \mathrm{~m}$ baseline.

We consider two different geometrical models for a single source: a Gaussian distribution and a uniform disk (UD). The results can be seen in Fig. 2. All curves show an increasing size of the object with increasing wavelength, as expected because of the different dust temperatures in different parts of the dust distribution. For the measurement performed with the $90 \mathrm{~m}$ baseline, the size varies between 16 and 30 mas for the Gaussian model

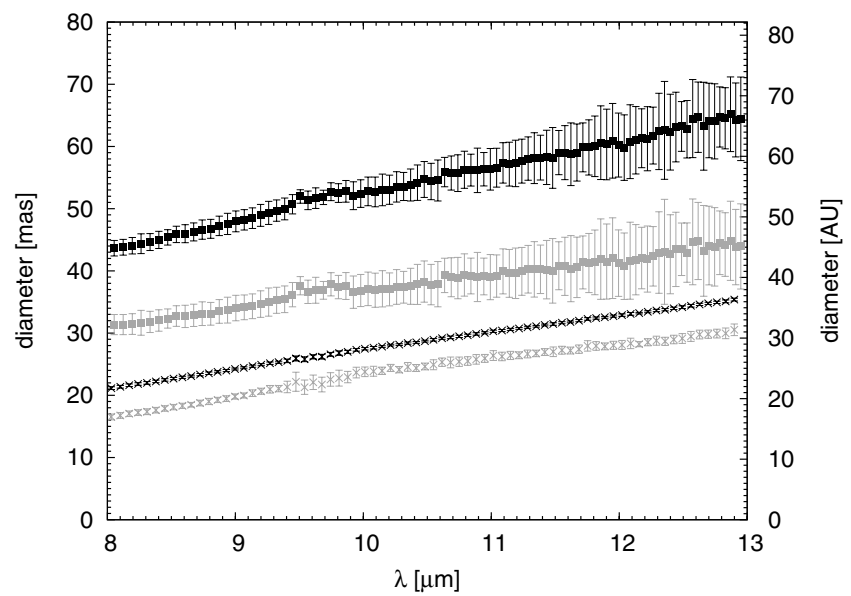

Fig. 2. Mid-infrared diameter of NGC 2264 IRS 1 as a function of wavelength for the 2005 data (crosses) measured with a $90 \mathrm{~m}$ baseline and the 2009 data (rectangulars) measured with a $40 \mathrm{~m}$ baseline. The values for the diameter in $\mathrm{AU}$ (right $y$-axis) were computed using a distance of 913 pc. Two different models were used: a Gaussian model (black) and a uniform disk model (gray).

and between 20 and 35 mas for the uniform disk model, whereas for the $40 \mathrm{~m}$ baseline, the size of the object lies between 30 and 44 mas for the Gaussian model and between 44 and 64 mas for the uniform disk model. As the observations were taken at different position angles, the different size estimations suggest an elongation in the dust distribution (e.g., a circumstellar disk or a flattened envelope). Although the intensity profile of the real source possibly differs from those distributions, the Gaussian model in particular is usually a good approximation of the midinfrared morphology of YSOs. Despite all the uncertainties in the visibility measurements, hence the size estimation, the fitting of the Gaussian model still helps us identify an asymmetry with a significance of $3 \sigma$ and the UD model within $2 \sigma$. This result clearly suggests that the MIR emission region is not spherically symmetric but elongated.

\subsection{Temperature gradient model}

Many young stars of both low and high mass are known to have circumstellar disks (e.g., Kraus et al. 2010; Leinert et al. 2004; Ratzka et al. 2009). As a first approach to interpreting 

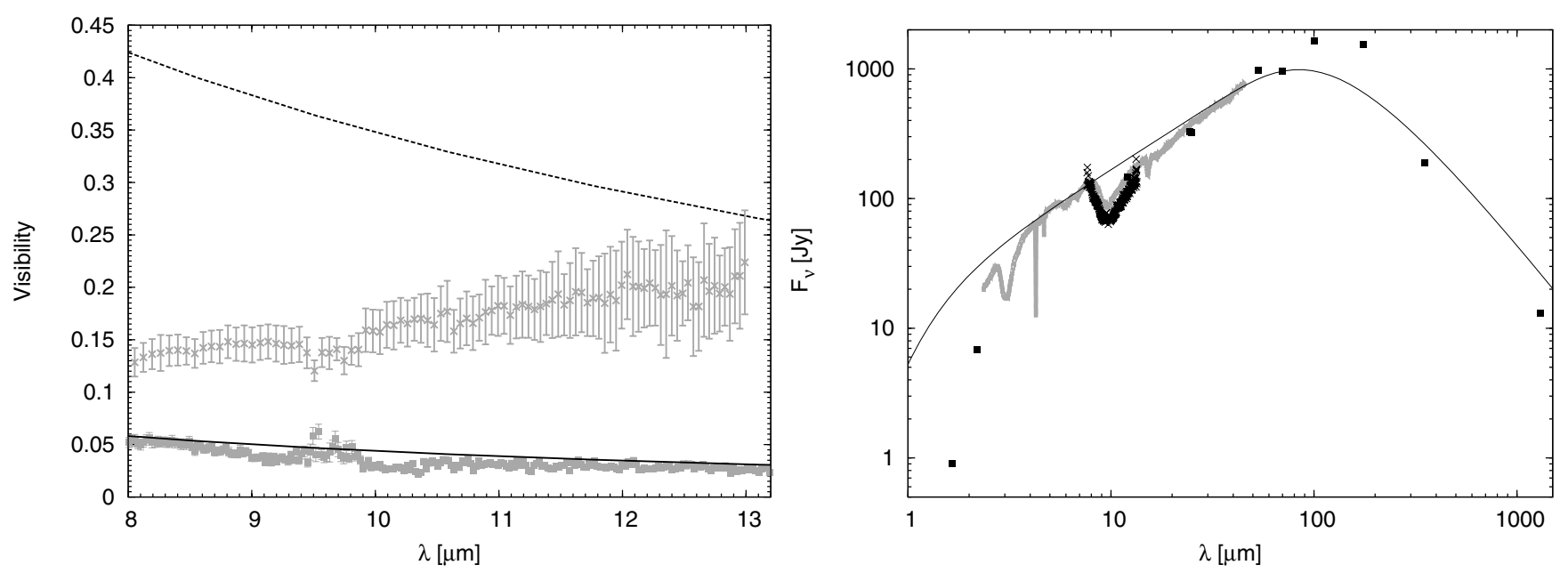

Fig. 3. Left: comparison of the best-fit temperature-gradient model to the visibilities of NGC 2264 IRS 1 with the model parameters described in Sect. 3.2 (crosses for the $40 \mathrm{~m}$ baseline, rectangulars for the $90 \mathrm{~m}$ baseline). The resulting modeled visibility for the $40 \mathrm{~m}$ baseline is shown by the black dashed line, the one for the $90 \mathrm{~m}$ baseline by the black solid line. Right: comparison of the SED of our best-fit temperature-gradient model (black line) described in Sect. 3.2 with the ISO spectrum (gray line) of NGC 2264 IRS 1. The MIDI spectrum is shown with black crosses, all other measurements from Table 1 are shown with black rectangulars.

simultaneously SED and N-band spatial data, we therefore use a temperature-gradient model for a circumstellar disk as described in Malbet et al. (2005). The disk specified by this model is optically thick, but geometrical thin with an inner radius $r_{\min }$ and an outer radius $r_{\max }$. The distribution of the temperature in the disk is described following the equation

$T(r)=T_{0}\left(\frac{r}{r_{0}}\right)^{-q}$,

where $T_{0}$ is the temperature at a reference radius $r_{0}=1 \mathrm{AU}$. The exponent $q$ is expected to lie approximately between 0.5 for passive, irradiated, flared disks and 0.75 for passive, irradiated, flat disks (Malbet et al. 2005).

One can imagine such a disk to be composed of rings with radius $r$ and thickness $d r$. If every ring emits blackbody radiation, one can evaluate the flux by integrating over the radius $r$ such that

$F_{\lambda}(0)=\frac{2 \pi}{d^{2}} \int_{r_{\min }}^{r_{\max }} r B_{\lambda}(T(r)) \cos (i) \mathrm{d} r$

where $d$ is the distance to the object, $B_{\lambda}$ the Planck function for a blackbody with temperature $T(r)$, and $i$ the inclination of the disk. For the visibility, one then gets

$V_{\lambda}(b, 0)=\frac{2 \pi}{F_{\lambda}(0) d^{2}} \int_{r_{\min }}^{r_{\max }} r B_{\lambda}(T(r)) J_{0}\left(2 \pi b \frac{r}{d}\right) \mathrm{d} r$

where $J_{0}$ is the Bessel function of zeroth order and $b$ the baseline according to

$b=\sqrt{u_{\Theta}^{2}+v_{\Theta}^{2} \cdot \cos ^{2}(i)}$.

Here, $\Theta$ is the position angle of the disk and $u_{\Theta}$ and $v_{\Theta}$ are given by

$u_{\Theta}=u \cos \Theta-v \sin \Theta$

and

$v_{\Theta}=u \sin \Theta+v \cos \Theta$.
We calculated a large number $(\approx 1000)$ of temperature-gradient models and tried to find a model that is simultaneously able to reproduce all observations (both visibilities and SED). For this, we varied the inner radius between 0.5 and $2 \mathrm{AU}$ (in steps of $0.5 \mathrm{AU}$ ) and $T_{0}$ between 1400 and $3000 \mathrm{~K}$ in steps of $200 \mathrm{~K}$. For the parameter $q$, we tested the values $0.5,0.6$, and 0.7 . Inclination and position angle were varied in steps of $10^{\circ}$.

The temperature gradient model with the parameters listed below is able to reproduce the SED in the mid- and far-infrared, but fails in the NIR. All of the calculated models failed completely to reproduce the shape of the visibility measured with the $40 \mathrm{~m}$ baseline (rising values going to larger wavelengths). As the best-fit model, we therefore show the model that most successfully reproduces the other visibility curve and the SED simultaneously, although different models led to a better fit for the $40 \mathrm{~m}$ visibility at least with respect to its absolute level. The best-fit model is shown in Fig. 3 and has the following parameters:

$$
\begin{aligned}
& -r_{\min }=0.5 \mathrm{AU} ; \\
& -r_{\max }=4000 \mathrm{AU} ; \\
& -T_{0}=2350 \mathrm{~K} ; \\
& -i=75^{\circ} ; \\
& -q=0.5 ; \\
& -\Theta=90^{\circ} .
\end{aligned}
$$

The spectral features seen in the SED and the effects of the silicate absorption on the visibility cannot be reproduced, as radiative transfer effects (emission, absorption, and scattering) are not included in the model.

A temperature of $T_{0}=2350 \mathrm{~K}$ at a reference radius of $1 \mathrm{AU}$ would imply a temperature of $3250 \mathrm{~K}$ at the inner disk radius of $0.5 \mathrm{AU}$ for this model. This is much too hot for the existence of normal dust grains. A possible explanation could be the existence of an inner gas disk (Kraus et al. 2008) or of dust grains with higher sublimation temperature (Benisty et al. 2010).

\subsection{Disk and envelope models in the Robitaille grid}

To check a wider parameter space for different models (especially for the possibility of a circumstellar envelope), we employed the online SED fitting tool developed by 

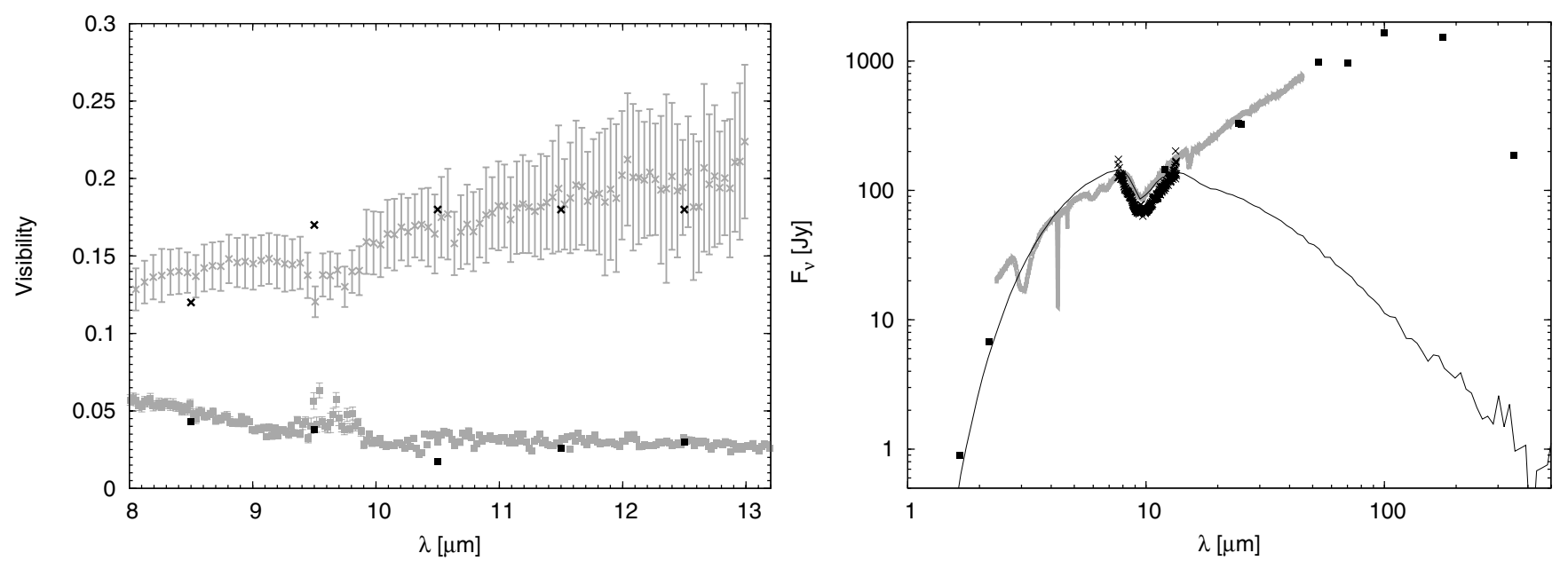

Fig. 4. Left: comparison of the best-fit Robitaille disk model (black crosses $40 \mathrm{~m}$ baseline, black rectangulars $90 \mathrm{~m}$ baseline) for the visibilities of NGC 2264 IRS 1 with the MIDI visibilities (gray rectangulars $90 \mathrm{~m}$ baseline, gray crosses $40 \mathrm{~m}$ baseline). Right: comparison of the best-fit Robitaille model (black line) for the spectrum of NGC 2264 IRS 1 with the ISO spectrum (gray line), the MIDI spectrum (black crosses), and the data points from Table 1 (black rectangulars).

Robitaille et al. (2007). This tool compares 200000 models of the SEDs of YSOs, which were precomputed using a 2D radiative transfer code (Whitney et al. 2003), with the measurements. These models are described by a set of 28 parameters, such as the properties of the central object (e.g., mass, temperature), the properties of the circumstellar envelope (e.g., outer radius, accretion rate, opening angle), the circumstellar disk (e.g., mass, outer disk radius, flaring), and the ambient density (for details see Robitaille et al. 2006). In addition to defining SED points, the user has to set a range for the interstellar extinction and for the distance of the object. We chose the interstellar extinction $A_{V}$ to be between 0 and 40 mag and the distance $d$ to be between $800 \mathrm{pc}$ and $1000 \mathrm{pc}$.

The number of points that can be fitted by the SED online tool is limited. Therefore, from the ISO spectrum about one flux point per one micron was used. From the MIDI spectrum, about two flux points per micron were chosen as the shape of the spectrum changes much more in this wavelength regime because of the silicate absorption feature.

When comparing the model visibilities and fluxes to the observed data, it is important to consider the very different (effective) beam-sizes of the different observations. The MIDI instrument has an effective field-of-view of $0.5^{\prime \prime}$ (slit width), corresponding to $\approx 450 \mathrm{AU}$ at the distance of our target. The beam sizes for all other data points beyond NIR wavelengths are much larger: the beam size from which the ISO spectrum was extracted $(\approx 13000 \times 18000 \mathrm{AU})$ is already $\approx 1100$ times larger, and the beam sizes for all far-infrared data points are at least 3000 times larger than the MIDI field-of-view. The ISO and far-infrared fluxes do therefore not only trace the emission from the central YSO and its immediate circumstellar material (on spatial scales of up to 500 AU), but also contain contributions from the surrounding extended molecular cloud, on spatial scales of $\gtrsim 20000 \mathrm{AU}(\widehat{=} \gtrsim 0.1 \mathrm{pc}$ ).

Therefore, as a first approach we include all SED points with $\lambda>13 \mu \mathrm{m}$ only as upper limits. To compute the visibilities, we used the HO-CHUNK code from Whitney et al. (2003) to calculate images for the model. Doing so, the ten best-fit models consist of circumstellar disks only (see Sect. 3.3.1). As a second approach, none of the data points was used as an upper limit, but they all were given with their errors. Here, rather different best-fit models are found by the fitting tool. They all consist of a large circumstellar envelope and either have no additional disk, or just a small ring-like structure with radii from 0.5 to $8 \mathrm{AU}$ (see Sect. 3.3.2). For similar applications of this fitting tool and its advantages and limitations in this respect, we refer to for instance Linz et al. (2009), de Wit et al. (2010), and Follert et al. (2010). We note that the parameter space is not covered uniformly. In particular, the spacing of the grid parameters is much finer in the case of low-mass YSOs than for MYSOs.

\subsubsection{Robitaille disk models}

The SED and visibilities of the best-fit Robitaille disk models are shown in Fig. 4. This model is able to reproduce the flux in the near- and mid-infrared. To reproduce the flux in the FIR, one would have to add for example some blackbody components as shown later for the RADMC model (see Sect. 3.4). The model can reproduce the level of the $90 \mathrm{~m}$ baseline visibility, and also the $40 \mathrm{~m}$ baseline visibility is reproduced within the errorbars. The parameters of this best-fit model are (model ID 3004478):

$$
\begin{aligned}
& -M_{\text {star }}=11.6 M_{\odot} ; \\
& -T_{\text {star }}=27720 \mathrm{~K} ; \\
& -L_{\text {star }}=1.21 \times 10^{4} L_{\odot} ; \\
& -r_{\text {out }}=84 \mathrm{AU} ; \\
& -h_{\text {disk }}(100 \mathrm{AU})=5.6 \mathrm{AU} ; \\
& -m_{\text {disk }}=0.09 M_{\odot} ; \\
& -i=70^{\circ} \\
& -A_{V}=20.27 \mathrm{mag} ; \\
& -\Theta=40^{\circ}
\end{aligned}
$$

Here, $r_{\text {out }}$ is the outer disk radius and $h_{\text {disk }}$ would be the height of the disk when extending it to a radius of $100 \mathrm{AU}$.

\subsubsection{Envelope plus disk models}

The Robitaille envelope model with the smallest $\chi^{2}$ is shown in Fig. 5. It fails to model especially the shape of the $40 \mathrm{~m}$ baseline visibility curve. The flux in the mid-IR can be reproduced, but the model cannot reproduce the flux in the near-IR. To attain the flux level in the far-IR, one would once again have to add, e.g., a blackbody component. The parameters of the best-fit model are (model ID 3019090):

- $M_{\text {star }}=8.5 M_{\odot}$; 

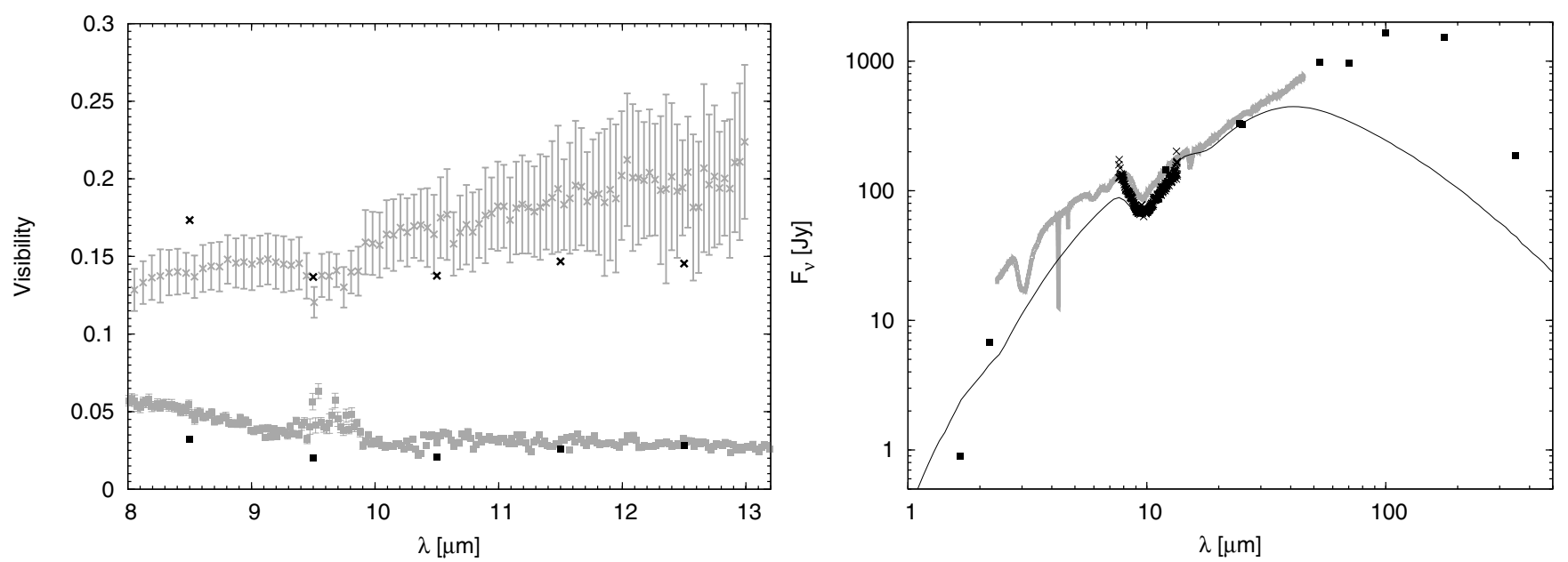

Fig. 5. Left: comparison of the best-fit Robitaille envelope model (black crosses $40 \mathrm{~m}$ baseline, black rectangulars $90 \mathrm{~m}$ baseline) for the visibilities of NGC 2264 IRS 1 with the MIDI visibilities (gray rectangulars $90 \mathrm{~m}$ baseline, gray crosses $40 \mathrm{~m}$ baseline). Right: comparison of the best-fit Robitaille model (black line) for the spectrum of NGC 2264 IRS 1 with the ISO spectrum (gray line), the MIDI spectrum (dark gray crosses), and the data points from Table 1 (black rectangulars).

- $T_{\text {star }}=4200 \mathrm{~K}$

- $R_{\text {star }}=82 R_{\odot}$;

- $L_{\text {star }}=2.05 \times 10^{3} L_{\odot}$;

$-r_{\text {envelope }}=36000 \mathrm{AU}$

- $m_{\text {envelope }}=1.84 M_{\odot}$;

$-r_{\text {disk }}=3.6 \mathrm{AU}$

- $h_{\text {disk }}(100 \mathrm{AU})=7.7 \mathrm{AU}$;

- $m_{\mathrm{disk}}=0.2 M_{\odot}$;

- $i=18^{\circ}$;

- $A_{V}=3.8 \mathrm{mag}$.

A change in the position angle does not in this case lead to significant differences for this model, as the dust distribution is nearly symmetric. Therefore, a best-fit position angle cannot be given. As mentioned before, this model consists mainly of a circumstellar envelope, with an outer radius of $r_{\text {envelope }}$. However, there is also a disk component with a very small outer radius $r_{\text {disk }}=3.6 \mathrm{AU}$. The central object is much cooler and larger than the central object in the disk models.

\subsection{Two-dimensional radiative transfer modeling}

A more detailed physical model for a circumstellar disk is obtained by using the two-dimensional (2-D) radiative transfer model code RADMC (Version 3.1, Dullemond \& Dominik 2004), which provides calculations of continuum radiative transfer in three-dimensional axisymmetric (therefore 2-D) circumstellar dust distributions around a central illuminating star. The main radiative transfer calculations are done with a Monte Carlo algorithm. To produce spectra and images, a post-processing with a ray-tracing code is done. The disk is described using a passive irradiated flared disk based on the model of Chiang \& Goldreich (1997), but the code also allows the user to insert a vertical puffed-up inner rim to produce a self-shadowed disk. The space is mapped in spherical coordinates. We therefore have radial grid points $r$ and angular grid points $\theta$, where $\theta=0$ is the polar axis and $\theta=\theta_{\max }=\frac{\pi}{2}$ is the equatorial plane. We again tried to reproduce the SED and visibilities simultaneously and also to fit the silicate absorption feature. To scan the parameter space, more than 10000 different models were calculated and compared to the observed data.

The geometry of the disk is described by the pressure scale height $h_{\text {disk }}$ at a reference radius $r_{\text {disk }}$, and an exponent $p$ in the following way (for $r_{\text {in }}<r<r_{\text {disk }}$ ):

$h(r)=h_{\text {disk }}\left(\frac{r}{r_{\text {disk }}}\right)^{p}$.

Furthermore, either the surface density at the outer radius $\sigma_{0}$ can be given by the user directly, or (as we did) be calculated from the user-defined total disk mass $m_{\text {disk }}=m_{\text {gas }}+m_{\text {dust }}$. We used a gas-to-dust ratio of 100 . The surface density as a function of radius is then given by

$\sigma(r)=\sigma_{0}\left(\frac{r}{r_{\text {disk }}}\right)^{-q}$

For $r>r_{\text {disk }}$, the exponent $q$ is chosen to be equal to 12 such that the density drops so fast that $r_{\text {disk }}$ is effectively the outer disk radius. This outer radius was varied between 30 AU and 150 AU. The density distribution in the whole disk is described by

$\rho(r, \theta) \propto \frac{\sigma(r)}{h(r) r} \exp \left[-\frac{1}{2}\left(\frac{\theta_{\max }-\theta}{\mathrm{h}(\mathrm{r})}\right)^{2}\right]$.

Parameters that remained fixed during the modeling process, were the temperature of the central object $T_{\text {star }}=25000 \mathrm{~K}$ (according to the spectral type B0-B2), the exponent $q=12$ for $r>r_{\text {disk }}$, and the temperature at the inner $\operatorname{rim} T_{\text {in }}=1700 \mathrm{~K}$. For the chemical composition of the dust, the optical properties of astronomical silicate (Draine \& Lee 1984) and graphite were chosen, using the grain size distribution of the MRN model (Mathis et al. 1977). A mixture of $70 \%$ silicate and 30\% graphite was assumed.

In addition to the original RADMC code, we introduced foreground extinction $A_{V}$, as otherwise all models failed to reproduce the deep silicate absorption feature. Parameters that were varied during the fitting process are shown in the following list together with their values for the best-fit RADMC model:

$-r_{\text {disk }}=50 \mathrm{AU}$

- $h_{\text {disk }}=0.1$

- $L_{\text {star }}=3.98 \times 10^{3} L_{\odot}$;

- $p=2 / 7$

- $m_{\text {disk }}=0.1 M_{\odot}$;

$-q=2.5$ for $r<r_{\text {disk }}$; 

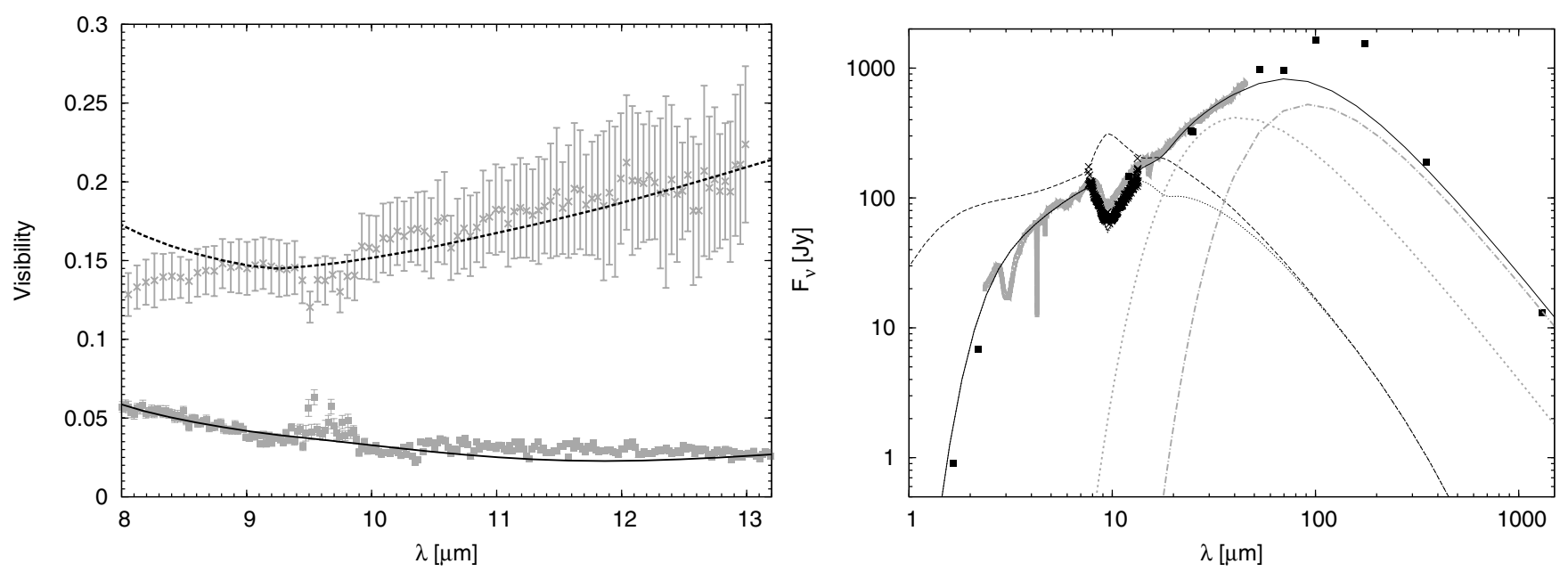

Fig. 6. Left: comparison of the best-fit RADMC model (dashed black line $40 \mathrm{~m}$ baseline, solid black line $90 \mathrm{~m}$ baseline) for the visibilities of NGC 2264 IRS 1 with the MIDI visibilities (rectangulars $90 \mathrm{~m}$ baseline, crosses $40 \mathrm{~m}$ baseline). Right: comparison of the best-fit RADMC model for the spectrum of NGC 2264 IRS 1 with the ISO spectrum (gray line), the MIDI spectrum (black crosses), and the data points from Table 1 (black rectangulars). The dashed black line is the RADMC model without foreground extinction, the dotted black line the model with a foreground extinction of $27 \mathrm{mag}$, and the solid black line the RADMC model with extinction and two additional blackbody components with temperatures of $55 \mathrm{~K}$ (gray dash-dotted line) and $120 \mathrm{~K}$ (gray dotted line).

$-i=30^{\circ}$

- $P A=40^{\circ}$

$-A_{V}=27 \mathrm{mag}$.

This best-fit model is shown in Fig. 6. It provides a good fit to the visibilities as well as the SED up to $12 \mu \mathrm{m}$.

The RADMC model describes the central YSO and its circumstellar matter up to the outer disk radius of $50 \mathrm{AU}$, i.e. predicts the emission in the central $100 \mathrm{AU}$ diameter region. Since this area is completely inside the MIDI field-of-view, the model visibility can be directly compared to the observed visibilities. For the fits to the SED, however, we have to take into account that the very large beams of the far-infrared observations must clearly include emission from the surrounding large-scale cloud, on spatial scales of $\sim 20000 \mathrm{AU}(\sim 0.1 \mathrm{pc})$ and larger, which is far outside the $r=50 \mathrm{AU}$ model area. This large-scale cloud emission should not be confused with a possible circumstellar envelope, but represents the molecular clump and the surrounding cloud in which IRS 1 is embedded (see discussion below). The mm-maps presented by Schreyer et al. (1997) show that the size of this cloud is about $1^{\prime}$ (corresponding to $\sim 55000 \mathrm{AU}$ or $\sim 0.27 \mathrm{pc})$.

The effects of this large-scale cloud are as follows: our MIDI measurements are insensitive to this large-scale emission, because it is far more extended than the angular resolution (and also the field-of-view) of MIDI. Since the large-scale cloud emission is completely over-resolved for MIDI, it will not affect the observed visibilities. The observed fluxes, however, are expected to be strongly affected by the large-scale cloud. First, the cloud material in front of the embedded object IRS 1 will cause considerable extinction. This effect is represented by the foreground extinction we use in our modeling. The second effect is that the cool dust in the large-scale cloud will produce far-infrared and mm-emission. Owing to the large beam sizes of the far-infrared and mm-observations, a large fraction of the observed fluxes in these beams will result from the large-scale cloud, and not from the central embedded object IRS 1. This explains why our RADMC model fluxes at wavelengths $\gtrsim 20 \mu \mathrm{m}$ are considerably lower than the observed fluxes. To approximately include this large-scale cloud emission component in our model, we added to our SED model in Fig. 6 two additional blackbody components. For the temperature of the first component, we used $T=55 \mathrm{~K}$, as determined by Schreyer et al. (1997) for the clump in which IRS 1 is embedded. For the second component, we used a temperature of $120 \mathrm{~K}$, which is typical of warm dust around massive YSOs in so-called "hot-cores" (Herbst \& van Dishoeck 2009). Fig. 6 shows that with the addition of these two components good agreement between the observed fluxes and the model can be achieved.

An interesting question is whether this $120 \mathrm{~K}$ component could be considered as an envelope around the disk, as for one interpretation of hot cores as an infalling envelope undergoing an intense accretion phase (Osorio et al. 2009). The RADMC model of a disk without surrounding envelope provides a very good fit of both visibilities. Adding a spherical circumstellar envelope with a radius of $\leq 250 \mathrm{AU}$ would change the model visibilities significantly (by $>5 \%$ ) and lead to disagreement between the model and the data. On the other hand, a (more or less) homogeneous envelope with a radius of $>250$ AU would be overresolved for MIDI and not affect the visibilities. However, to reproduce the far-infrared fluxes in the SED, the radius of the $120 \mathrm{~K}$ emitting region has to be at least $\geq 400 \mathrm{AU}$ (in the limiting case of optically thick blackbody emission). Owing to the limited MIDI field of view, the difference between the ISO and the MIDI spectrum could result from such an extended structure. The question of whether an extended circumstellar envelope is present can thus not be answered by the available data. Whether the material in such a hypothetical envelope would be gravitationally bound and ultimately accrete onto the circumstellar disk, remains another open question.

\section{Discussion}

We now want to discuss our findings presented in Sect. 3. We first compare the size of the mid-IR emission region as calculated in Sect. 3.1 to the corresponding sizes of similar objects. We then discuss the radiative transfer models.

\subsection{Comparison to MIR sizes of other massive YSOs}

The near-IR sizes of the disks of most YSOs (in particular Herbig Ae stars) show a tight correlation with the luminosity 

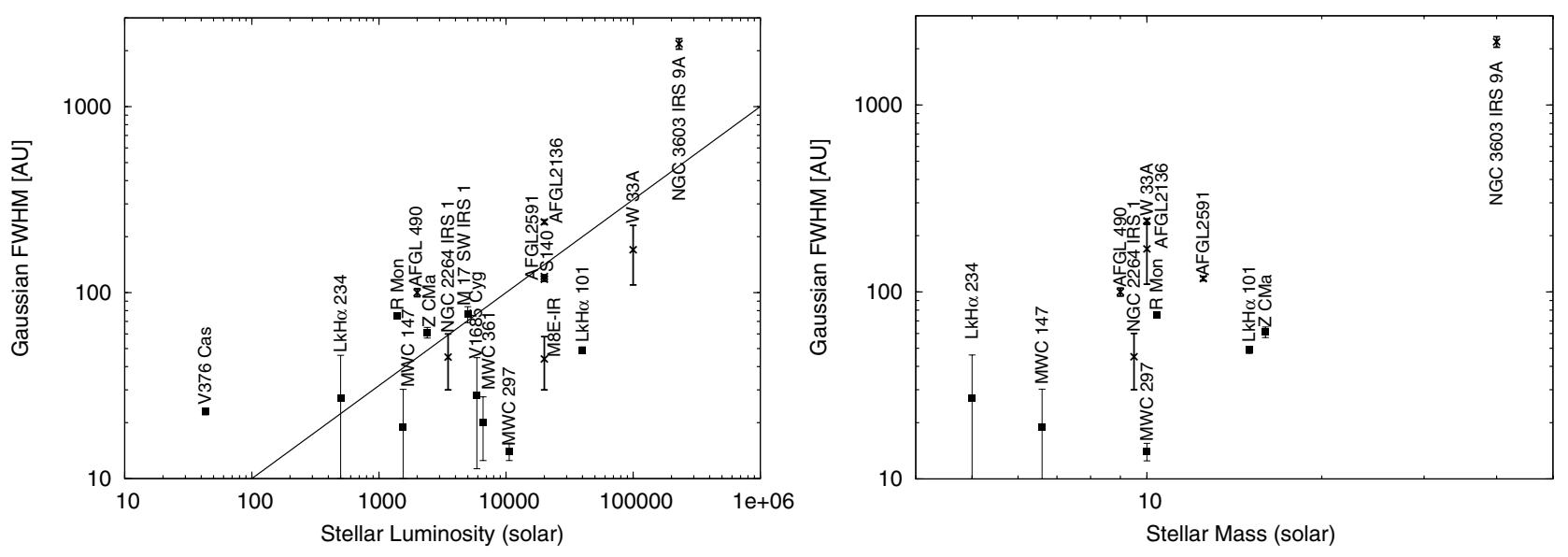

Fig. 7. Left: mid-infrared-size-luminosity diagram. Rectangulars correspond to Herbig Be stars, crosses to MYSOs. Values and references are shown in Table A.1. The black line corresponds to a $R \propto \sqrt{L}$ relation. Right: mid-infrared-size-stellar-mass diagram. Rectangulars correspond to Herbig Be stars, crosses to MYSOs.

(Monnier et al. 2005) because the emission in the NIR comes from regions close to the dust sublimation radius, which is sensitive to radiation from the central star and scales approximately with the square root of the luminosity. The mid-infrared emission, in contrast, originates from a wider range of regions: the hot inner wall located at the dust sublimation radius, the surface of an irradiated disk, and the circumstellar envelope. As shown in Monnier et al. (2009), the size of the region emitting in the MIR therefore spans a much larger region for a given luminosity. For circumstellar disks, factors such as the flaring of the disk and the shadowing caused by the inner rim play an important role. Here, we wish to compare the MIR size of NGC 2264 IRS 1 as found for the uniform disk model with the MIR sizes of other MYSOs, as well as with those of Herbig Be stars.

Only a few other MYSOs have been studied with high angular resolution in the MIR until now. Amongst them are AFGL 2591, AFGL 2136, AFGL 490, and S140 IRS 1 from the study of MIR sizes of Monnier et al. (2009), as well as W 33A (de Wit et al. 2007, 2010), and NGC 3603-IRS 9A (Vehoff et al. 2010). The sample of Herbig Be stars was also taken from Monnier et al. (2009). The disk size vs. luminosity diagram of these objects and NGC 2264 IRS 1, as well as the size vs. stellar mass diagram is shown in Fig. 7. Parameters such as stellar mass, distance, and the numbers for disk size and luminosity can be found in Table A.1. Both luminosity and mass of the central object seem to be correlated with the size of the MIR emission region. Owing to the factors considered above, objects with luminosities ranging between 1000 and $10000 L_{\odot}$ display a large scatter in their sizes (a factor of around 10). The same is true for the mass range of $\sim 8-20 M_{\odot}$. The size of the MIR emitting region around NGC 2264 IRS 1 seems to be typical for their mass and luminosity.

\subsection{Discussion of the radiative transfer models}

Models of a circumstellar envelope only did not provide good fits, as they were unable to reproduce visibilities and NIR flux simultaneously. Furthermore, the estimated sizes of the dust distribution (Sect. 3.1) are different for different position angles, which implies that there is an asymmetric dust distribution.

The radiative transfer modeling performed with RADMC suggests that IRS 1 has an optical thick, but geometrically thin, flaring circumstellar disk with a mass of $0.1 M_{\odot}$. This is supported by the results of the SED fitting process using the
Robitaille grid. Not only the best-fit Robitaille disk model but also the ten best-fit models have parameters describing the central object and the circumstellar disk that compare very well with those of the best-fit model found using RADMC. However, the inclinations of the Robitaille models vary between $20^{\circ}$ and $70^{\circ}$. The temperature and mass of the central object are slightly higher in all of these disk models than the values used for the RADMC model.

The two blackbody components producing the far-IR flux for our best-fit RADMC model could originate from a large surrounding cloud or envelope, respectively, that would with the large angular resolution of MIDI only be seen as foreground extinction. This would agree with the large foreground extinction ( $>20 \mathrm{mag}$ ) as used in the best-fit RADMC and derived from the ten best-fit Robitaille disk models. Furthermore, previous publications have suggested that there is a large amount of foreground extinction with values between 20 mag (Thompson et al. 1998) and $35 \mathrm{mag}$ (Thompson \& Tokunaga 1978). The inclination of the best-fit RADMC model is $30^{\circ}$, but owing to the poor uvcoverage this value is poorly constrained. The position angle of the jet-like feature seen in the near-IR is $20^{\circ}$ (see Fig. 8), and the position angle in our best-fit disk model is $40^{\circ}$. This does not match perfectly, but the value for the position angle is also poorly constrained. However, an overall geometric picture of a moderately inclined disk seems reasonable.

\section{Summary and conclusions}

We have presented MIDI mid-infrared interferometric observations of the massive young stellar object NGC 2264 IRS 1. The observed visibilities provide no hint of multiplicity of the central source in the separation range from $\sim 30 \mathrm{AU}$ (resolution limit) to $\sim 230$ AU (slit width limit). Geometric models (Sect. 3.1) suggest a size of $\sim 30-60$ AU for the mid-infrared emission region. This value is in good agreement with the mid-infrared sizes of similar objects.

Our modeling shows that a simple temperature-gradient model for a circumstellar disk can reproduce the SED at midand far-IR wavelengths, but fails to reproduce the SED in the near-IR and the observed visibilities; this model can therefore be ruled out.

We have performed a detailed radiative transfer modeling of the observed SED and visibilities with the RADMC code. It suggests a scenario of a geometrically flat but optically thick 


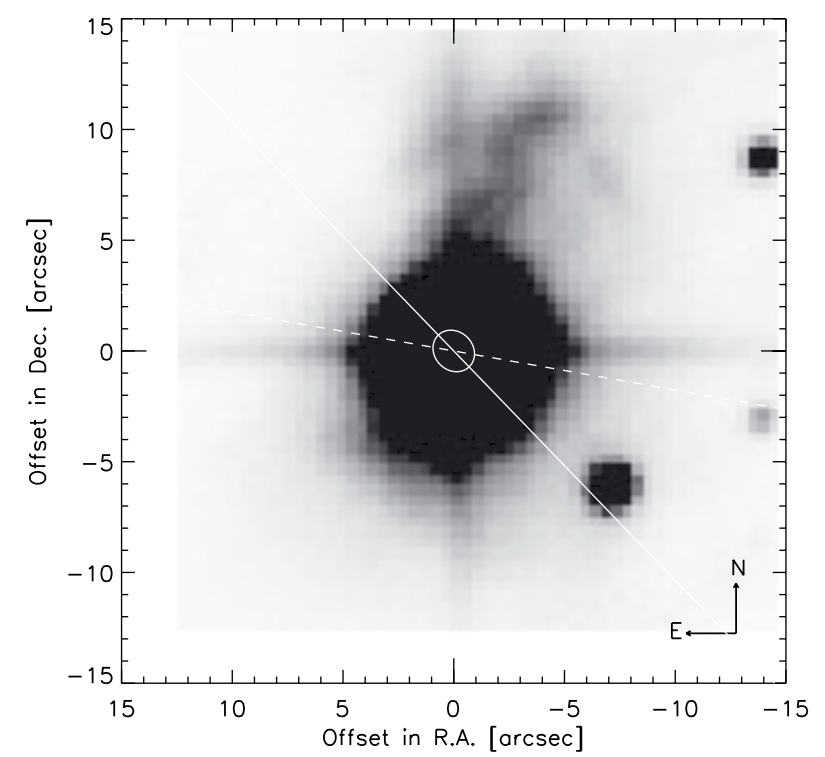

Fig. 8. K-band image of NGC 2264 IRS 1 from Schreyer et al. (1997). We overlay an ellipse (white) that shows a disk seen under an inclination of $30^{\circ}$ and a position angle of $40^{\circ}$. The size of the disk is not drawn to scale. The solid white line shows the orientation of the $40 \mathrm{~m}$ baseline, the dashed one the orientation of the $90 \mathrm{~m}$ baseline.

circumstellar dust disk. We also used the fitting tool by Robitaille et al. (2007), which leads to similar disk models and therefore confirms the results obtained with RADMC, whereas envelope models found within the Robitaille grid did not provide similarly good fits to SED and visibilities simultaneously. Although there is no observational evidence, the possible presence of a large $(r \geq 400 \mathrm{AU})$ spherical envelope around the disk cannot be excluded by the data. The mass of the circumstellar disk is about $0.1 M_{\odot}$. The data suggest an inclination angle of $\sim 30^{\circ}$ and a position angle of $\sim 40^{\circ}$ for the orientation of the disk. These values are consistent with an overall geometrical model based on the jet-like feature seen to the north-east of IRS 1. Comparing NGC 2264 IRS 1 to other YSOs, the size of its MIR emitting region seems to be typical of its luminosity and mass. However, the large scatter of sizes in this range of luminosities and masses points towards a wide variety of (disk) morphologies among these objects.

More observational research, such as future MIR interferometric observations, will provide tighter constraints on the circumstellar material around this interesting source and therefore will help us to clarify our understanding of the disks around high-mass YSOs.

Acknowledgements. We would like to thank the anonymous referee for his suggestions and comments that helped to improve the paper. We would also like to thank K. Dullemond for explanations and discussions about RADMC. We gratefully acknowledge funding of this work by the German Deutsche Forschungsgemeinschaft, DFG project number PR 569/8-1.

\section{References}

Acke, B., \& van den Ancker, M. E. 2004, A\&A, 426, 151 Acke, B., Verhoelst, T., van den Ancker, M. E., et al. 2008, A\&A, 485, 209 Allen, D. A. 1972, ApJ, 172, L55

Alonso-Albi, T., Fuente, A., Bachiller, R., et al. 2009, A\&A, 497, 117 Baxter, E. J., Covey, K. R., Muench, A. A., et al. 2009, AJ, 138, 963 Benisty, M., Natta, A., Isella, A., et al. 2010, A\&A, 511, A74 Berger, J. P., \& Segransan, D. 2007, New A Rev., 51, 576 Bonnell, I. A., \& Bate, M. R. 2006, MNRAS, 370, 488 Bonnell, I. A., Bate, M. R., \& Zinnecker, H. 1998, MNRAS, 298, 93
Cesaroni, R., Neri, R., Olmi, L., et al. 2005, A\&A, 434, 1039

Chiang, E. I., \& Goldreich, P. 1997, ApJ, 490, 368

Chini, R., Kruegel, E., \& Kreysa, E. 1986, A\&A, 167, 315

Crampton, D., \& Fisher, W. A. 1974, Publications of the Dominion Astrophysical Observatory Victoria, 14, 283

Cunningham, A., Krumholz, M. R., Klein, R. I., \& McKee, C. F. 2009, in Am. Astron. Soc. Meet. Abstr., 214, 604.05

de Wit, W. J., Hoare, M. G., Oudmaijer, R. D., \& Mottram, J. C. 2007, ApJ, 671, L169

de Wit, W. J., Hoare, M. G., Fujiyoshi, T., et al. 2009, A\&A, 494, 157

de Wit, W. J., Hoare, M. G., Oudmaijer, R. D., \& Lumsden, S. L. 2010, A\&A, 515, A45

Draine, B. T., \& Lee, H. M. 1984, ApJ, 285, 89

Drew, J. E., Busfield, G., Hoare, M. G., et al. 1997, MNRAS, 286, 538

Dullemond, C. P., \& Dominik, C. 2004, A\&A, 417, 159

Follert, R., Linz, H., Stecklum, B., et al. 2010, A\&A, 522, A17

Fuente, A., Rizzo, J. R., Caselli, P., Bachiller, R., \& Henkel, C. 2005, A\&A, 433, 535

Gibb, E. L., Whittet, D. C. B., Boogert, A. C. A., \& Tielens, A. G. G. M. 2004, ApJS, 151, 35

Guertler, J., Henning, T., Koempe, C., et al. 1996, A\&A, 315, L189

Harvey, P. M., Campbell, M. F., \& Hoffmann, W. F. 1977, ApJ, 215, 151

Herbig, G. H., Andrews, S. M., \& Dahm, S. E. 2004, AJ, 128, 1233

Herbst, E., \& van Dishoeck, E. F. 2009, ARA\&A, 47, 427

Hernández, J., Calvet, N., Briceño, C., Hartmann, L., \& Berlind, P. 2004, AJ, 127,1682

Kastner, J. H., Weintraub, D. A., \& Aspin, C. 1992, ApJ, 389, 357

Köhler, R., \& Jaffe, W. 2008, in The Power of Optical/IR Interferometry: Recent Scientific Results and 2nd Generation (Heidelberg: Springer), ed. A. Richichi, F. Delplancke, F. Paresce, \& A. Chelli, 569

Kraus, S., Preibisch, T., \& Ohnaka, K. 2008, ApJ, 676, 490

Kraus, S., Hofmann, K., Menten, K. M., et al. 2010, Nature, 466, 339

Kuiper, R., Klahr, H., Beuther, H., \& Henning, T. 2010, ApJ, 722, 1556

Leinert, C., Graser, U., Przygodda, F., et al. 2003, Ap\&SS, 286, 73

Leinert, C., van Boekel, R., Waters, L. B. F. M., et al. 2004, A\&A, 423, 537

Lester, D. F., Harvey, P. M., Joy, M., \& Ellis, Jr., H. B. 1986, ApJ, 309, 80

Linz, H., Henning, T., Feldt, M., et al. 2009, A\&A, 505, 655

Malbet, F., Lachaume, R., Berger, J., et al. 2005, A\&A, 437, 627

Mathis, J. S., Rumpl, W., \& Nordsieck, K. H. 1977, ApJ, 217, 425

McKee, C. F., \& Tan, J. C. 2003, ApJ, 585, 850

Men'shchikov, A. B., \& Henning, T. 1997, A\&A, 318, 879

Monnier, J. D., Millan-Gabet, R., Billmeier, R., et al. 2005, ApJ, 624, 832

Monnier, J. D., Tuthill, P. G., Ireland, M., et al. 2009, ApJ, 700, 491

Murakawa, K. 2010, A\&A, 522, A46

Murakawa, K., Preibisch, T., Kraus, S., et al. 2008, A\&A, 488, L75

Nakano, M., Sugitani, K., \& Morita, K. 2003, PASJ, 55, 1

Osorio, M., Anglada, G., Lizano, S., \& D’Alessio, P. 2009, ApJ, 694, 29

Ratzka, T., Schegerer, A. A., Leinert, C., et al. 2009, A\&A, 502, 623

Robitaille, T. P., Whitney, B. A., Indebetouw, R., Wood, K., \& Denzmore, P. 2006, ApJS, 167, 256

Robitaille, T. P., Whitney, B. A., Indebetouw, R., \& Wood, K. 2007, ApJS, 169, 328

Sargent, A. I., van Duinen, R. J., Nordh, H. L., et al. 1984, A\&A, 135, 377

Schreyer, K., Helmich, F. P., van Dishoeck, E. F., \& Henning, T. 1997, A\&A, 326,347

Schreyer, K., Stecklum, B., Linz, H., \& Henning, T. 2003, ApJ, 599, 335

Schreyer, K., Semenov, D., Henning, T., \& Forbrich, J. 2006, ApJ, 637, L129

Schwartz, P. R., Thronson, Jr., H. A., Odenwald, S. F., et al. 1985, ApJ, 292, 231

Shevchenko, V. S., \& Yakubov, S. D. 1989, Soviet Ast., 33, 370

Sloan, G. C., Kraemer, K. E., Price, S. D., \& Shipman, R. F. 2003, ApJS, 147, 379

Thamm, E., Steinacker, J., \& Henning, T. 1994, A\&A, 287, 493

Thiebaut, E., Bouvier, J., Blazit, A., et al. 1995, A\&A, 303, 795

Thompson, R. I., \& Tokunaga, A. T. 1978, ApJ, 226, 119

Thompson, R. I., Corbin, M. R., Young, E., \& Schneider, G. 1998, ApJ, 492, L177

Tristram, K. R. W. 2007, Ph.D. Thesis, Max-Planck-Institut für Astronomie (Heidelberg)

van der Tak, F. F. S., van Dishoeck, E. F., Evans, II, N. J., Bakker, E. J., \& Blake, G. A. 1999, ApJ, 522, 991

Vehoff, S., Hummel, C. A., Monnier, J. D., et al. 2010, A\&A, 520, A78

Ward-Thompson, D., Zylka, R., Mezger, P. G., \& Sievers, A. W. 2000, A\&A, 355,1122

Whitney, B. A., Wood, K., Bjorkman, J. E., \& Wolff, M. J. 2003, ApJ, 591, 1049

Zinnecker, H., \& Bate, M. R. 2002, in Hot Star Workshop III: The Earliest Phases of Massive Star Birth (ASP: San Francisco), ed. P. Crowther, ASP Conf. Ser., 267, 209

Zinnecker, H., \& Yorke, H. W. 2007, ARA\&A, 45, 481 
A\&A 532, A109 (2011)

\section{Appendix A: Parameters and references for size-luminosity plot}

Table A.1. MIR-sizes of MYSOs and Herbig Be stars.

\begin{tabular}{lcccccc}
\hline \hline Object & $\begin{array}{c}\text { Diameter } \\
{[\mathrm{AU}]}\end{array}$ & $\begin{array}{c}\text { Distance } \\
{[\mathrm{pc}]}\end{array}$ & $\begin{array}{c}\text { Luminosity } \\
{\left[L_{\odot}\right]}\end{array}$ & $\begin{array}{c}\text { Mass } \\
{\left[M_{\odot}\right]}\end{array}$ & SpT & Reference \\
\hline NGC 3603 IRS 9A & 2177 & 7000 & $2.3 \times 10^{5}$ & 40 & & 1 \\
W 33A & $115-230$ & 3800 & $1 \times 10^{5}$ & 10 & & 2 \\
V376 Cas & 23 & 630 & 43 & & B5e & 3,4 \\
MWC 147 & 19 & 800 & 1550 & 6.6 & B6 & $3,5,6$ \\
R Mon & 75 & 800 & 1400 & 10.4 & B0 & $3,7,8$ \\
Z CMa & 61 & 1000 & 2400 & 16 & B & $3,9,10$ \\
MWC 297 & 14 & 250 & 10600 & 10 & B1.5 & $3,11,12$ \\
V1685 Cyg & 28 & 980 & 5890 & & B3 & 3,4 \\
MWC 361 & 20 & 440 & 6600 & & B2 & 3,4 \\
LkH $\alpha$ 234 & 27 & 1000 & 500 & 5 & B7 & $3,13,14$ \\
AFGL 490 & 100 & 1000 & $2 \times 10^{3}$ & $8-10$ & B2 & 3,15 \\
LkH $\alpha$ 101 & 49 & 700 & $2-6 \times 10^{4}$ & 15 & Be & 3,16 \\
S140 IRS 1 & 122 & 910 & $2 \times 10^{4}$ & & & $3,17,18$ \\
AFGL 2136 & 240 & 2000 & $2 \times 10^{4}$ & $>10$ & & 3,19 \\
AFGL 2591 & 118 & 1000 & $2 \times 10^{4}$ & $10-15$ & & 3,20 \\
M 17 SW IRS 1 & $69-84$ & 2100 & $5 \times 10^{3}$ & & B0 & 21 \\
M8E-IR & $30-57$ & 1500 & $2 \times 10^{4}$ & & B0 & 22 \\
NGC 2264 IRS 1 & $30-60$ & 913 & $1.5-4.7 \times 10^{3}$ & 9.5 & B0-B2 & see Chapt. 1, 3.1 \\
\hline
\end{tabular}

References. (1) Vehoff et al. (2010); (2) de Wit et al. (2007); (3) Monnier et al. (2009); (4) Acke \& van den Ancker (2004); (5) Kraus et al. (2008); (6) Hernández et al. (2004); (7) Murakawa et al. (2008); (8) Murakawa (2010); (9) Alonso-Albi et al. (2009); (10) Thiebaut et al. (1995); (11) Acke et al. (2008); (12) Drew et al. (1997); (13) Shevchenko \& Yakubov (1989); (14) Fuente et al. (2005); (15) Schreyer et al. (2006); (16) Herbig et al. (2004); (17) Crampton \& Fisher (1974); (18) Lester et al. (1986); (19) Kastner et al. (1992); (20) van der Tak et al. (1999); (21) Follert et al. (2010); (22) Linz et al. (2009). 